\title{
Björn-Olav Dozo, Anthony Glinoer, Michel Lacroix (a cura di), Imaginaires de la vie littéraire. Fiction, figuration, configuration
}

\section{Roberta Sapino}

\section{(2) OpenEdition}

\section{Journals}

\section{Edizione digitale}

URL: http://journals.openedition.org/studifrancesi/2331

DOI: 10.4000/studifrancesi.2331

ISSN: 2421-5856

\section{Editore}

Rosenberg \& Sellier

\section{Edizione cartacea}

Data di pubblicazione: 1 aprile 2014

Paginazione: 203-204

ISSN: 0039-2944

\section{Notizia bibliografica digitale}

Roberta Sapino, «Björn-Olav Dozo, Anthony Glinoer, Michel Lacroix (a cura di), Imaginaires de la vie littéraire. Fiction, figuration, configuration », Studi Francesi [Online], 172 (LVIII | I) | 2014, online dal 01 avril 2014, consultato il 18 septembre 2020. URL : http://journals.openedition.org/studifrancesi/2331 ; DOI : https://doi.org/10.4000/studifrancesi.2331

\section{Questo documento è stato generato automaticamente il 18 settembre 2020.}

\section{cc) $(9)$}

Studi Francesi è distribuita con Licenza Creative Commons Attribuzione - Non commerciale - Non opere derivate 4.0 Internazionale. 


\title{
Björn-Olav Dozo, Anthony Glinoer, Michel Lacroix (a cura di), Imaginaires de la vie littéraire. Fiction, figuration, configuration
}

\author{
Roberta Sapino
}

\section{NOTIZIA}

BJÖRN-OLAV DOZO, ANTHONY GLINOER, MICHEL LACROIX (a cura di), Imaginaires de la vie littéraire. Fiction, figuration, configuration, Rennes, Presses Universitaires de Rennes, 2012, pp. 378.

1 Escluso da tempo dal legittimo campo d'interrogazione degli studi letterari, l'autore ha recentemente fatto ritorno nel discorso critico. Lungi dal significare un ritorno al passato, il superamento della teoria letteraria basata sulla morte dell'autore non segna tanto la nascita di una nuova teoria a essa contrapposta, quanto piuttosto lo sviluppo di un cantiere di ricerca che, se da un lato prende le distanze dalle affermazioni di Barthes e Foucault, dall'altro dimostra di porre su di esse le sue basi.

2 I numerosi contributi raccolti in questo volume si propongono di offrire una panoramica di ampio respiro su una riflessione collettiva in grande fermento ma ancora, come osserva Michel LACRoIX nell'introduzione (pp. 7-22), priva di una sua sistematicità. Ne risulta un percorso ricco e variegato, cronologicamente esteso dal XIX al XXI secolo, che non esita a superare i confini dell'esagono per spingersi in Canada e negli Stati Uniti, né a discostarsi a tratti dalla letteratura "consacrata" per avventurarsi in territori raramente esplorati come quelli della chick lit. Pur nella sua varietà, il corpus analizzato rivela un comune interesse per l'autore in quanto figura chiave di determinate dinamiche sociali e testuali, in continua e feconda relazione con un mondo letterario che si impone soprattutto come luogo di socializzazione e di definizione identitaria. Si delinea così un'immagine dell'autore in società che, afferma Jean-Pierre 
BERTRAND nel saggio di ouverture («Le comble de la vie littéraire: Paludes», pp. 23-32), molto deve all'operazione di rottura compiuta da André Gide in Paludes, ironica sovversione del mito dello scrittore condannato alla solitudine e all'insoddisfazione.

3 Il volume è suddiviso in quattro sezioni, la prima delle quali, intitolata «Personnages génériques et écrivains imaginaires», propone una rassegna di figure autoriali fittizie molto lontane dall'aura di sacralità del grantécrivain. Ad aprire il discorso è Valérie STIÉNON (L'homme de lettres en personnage générique. La sociologie-fiction des «physiologies», pp. 35-46), che si concentra sul genere della physiologie littéraire, molto in voga durante la Monarchia di Luglio, per mostrare come la rappresentazione dello scrittore in quanto personnage générique permetta non solo di fornirne una visione anti-eroica in netto contrasto con la tendenza dell'epoca, ma anche di porre le basi di un discorso destinato a concretizzarsi nell'estetica realista. Dopo aver ripercorso lo sviluppo della figura del rond-de-cuir nella letteratura europea e francese fino al suo âge d'or di inizio novecento, Cyril PIROUX (Le rond-de-cuir dans le roman français: figure symbolique de l'écrivain moderne?, pp. 47-62) ipotizza che in essa si manifesti una vera e propria crisi autoproclamata del romanzo e dello statuto di scrittore; a seguire, Martine-Emmanuelle LAPOINTE (Figures de l'engagement politique dans trois romans québécois contemporains, pp. 63-74) si chiede se nella letteratura quebecchese contemporanea ci sia ancora spazio per la scrittura engagée, suggerendo che essa compensi l'evidente disillusione col desiderio di inscriversi in una storia comunitaria e di costituire una sorta di lascito per i lettori e le generazioni future. Marie-Pier LUNEAU (Talons aiguilles, best-sellers et milieu de l'édition: figurations du personnel littéraire en chick lit, pp. 75-90) ci trasporta nel mondo della chick lit e fa notare come, pur rappresentando figure e dinamiche editoriali radicalmente opposte a quelle della letteratura canonizzata, le autrici dei romanzi presi in analisi non aspirino a mettere in discussione l'istituzione letteraria, ma piuttosto a trovare un loro spazio, seppur problematico, di legittimazione. Infine, Charline PLUVINET ( «Pataulogie» de la littérature: l'écrivain afro-américain à l'épreuve de la fiction dans "Erasure" de Percival Everett, pp. 91-103) indaga i meccanismi attraverso i quali Percival Everett scardina dall'interno un sistema spesso propenso ad attribuire identità autoriali più in virtù delle origini dello scrittore che della tradizione culturale nella quale la sua opera effettivamente si inserisce.

4 La seconda sezione, «Personnages légendaires et histoire littéraire», si concentra sull'inscrizione di figure di spicco della vita letteraria all'interno della finzione romanzesca. Vincent LAISNEY (Enquête préliminaire sur l'évolution littéraire. Le Termite de J.-H. Rosny (1889), pp. 107-122) propone di leggere Le Termite non tanto come una storiografia romanzata del cenacolo dei Goncourt e della sociabilité littéraire dell'epoca, quanto piuttosto come una satira dell'estetica naturalista, attraverso la quale Rosny mira a far vacillare il gruppo già costituito per acquisirne la direzione spirituale e indirizzarne il percorso verso nuove strade. Altro roman cénaculaire, Le Soleil des Morts di Camille Mauclaire, analizzato da Pascal DURAND (Le dernier Mardi et le dernier cénacle. De Calixte Armel à Mallarmé, pp. 123-136), si rivela una critica piuttosto radicale dei Mardis di Mallarmé, ancor più aspra perché avanzata da un membro interno al circolo. Con Pascal BRISSETTE (Visite au grand écrivain et récitation d'outre-tombe: Nelligan dans Le beau risque, pp. 137-152) si torna in Québec per assistere a una scène de récitation in cui il poeta Émile Nelligan, ormai anziano e lontano dalla vita letteraria, incarna un'identità nazionale e letteraria tanto minacciata quanto resistente; David VRYDAGHS ( Le surréalisme fictif. Motifs critiques et réécritures historiographiques dans Odile, Gilles et 
Aurélien, pp. 153-166) si concentra invece su tre rappresentazioni del movimento surrealista che, con un'acuta postura critica e un tono talvolta dissacrante, invitano a rivalutarne la posizione all'interno del panorama della storiografia letteraria. JeanPhilippe MARTEL (Roger Nimier, personnage(s) de roman: scénographies d'un hussard de papier, pp. 167-184) chiude la sezione mostrando come la persona di Roger Nimier, inserita in opere di finzione, assuma una funzione legittimante nei confronti di determinati discorsi sulla letteratura e sullo statuto dello scrittore in società.

In «Médiateurs et médiatisations» il focus si estende su quei personaggi che, pur non essendo scrittori, influenzano le dinamiche della sociabilité littéraire. Guillaume MCNEIL ARTEAU (Le journaliste chez Balzac, ou le libéralisme dans les lettres, pp. 187-202) e Guillaume PINSON (De Lucien de Rubempré à Gédéon Spilett ou de l'écrivain-journaliste au reporter qui n'écrit plus: scénarios et contre-scénarios, pp. 203-216) si concentrano sulla figura del giornalista, il primo per offrire una lettura politica del balzachiano Illusions Perdues e mettere in luce i legami tra l'azione "corrosiva" della stampa a livello nazionale e la sterilità creativa dell'individuo, il secondo per sottolineare come il roman du reporter, sviluppatosi alla fine del XIX secolo, non esiti ad appropriarsi delle forme estetiche e relazionali tipiche del giornalismo, affrancandosi dalle reticenze e dai sospetti tipici del roman du journaliste. Björn-Olav DOzo e Michel LACROIX («Le lieu géométrique de toutes les agitations»: écrivains fictifs et prix littéraires, de Renée Dunan à Vincent Ravalec, pp. 217-236) si chiedono in che misura e con quali esiti i premi letterari entrino nella finzione romanzesca, mentre Sylvie DUCAs (Fables contemporaines du «bibliothécaire en morceaux» ou l'auteur bibliophobe et l'a-bibliothèque, pp. 237-250) ipotizza che la rappresentazione spesso poco lusinghiera - della figura del bibliotecario rifletta la difficoltà per l'autore di ricavarsi, e mantenere, il proprio spazio ideale nel mondo letterario. Il traduttore, portatore di riflessioni linguistiche, estetiche e identitarie, è al centro della ricerca di Patricia GODBOUT (De quelques personnages de traducteurs dans la littérature québécoise, pp. 251-260); nel saggio di Anne-Marie CLÉMENT (Représentations contemporaines de l'écrivain fictif dans ses habits de biographe, pp. 261-272) si discute invece della figura del biografo, vista come identità transitoria di un autore en devenir alla ricerca di legittimazione. Conclude Caroline PAQUETTE (Entre manuscrits et souffrance: l'éditeur artiste dans le roman québécois contemporain, pp. 273-284), mostrando come la rappresentazione dell'editoreartista, al quale sono attribuite caratteristiche normalmente proprie dell'autore, metta in discussione la possibilità di successo della fragile relazione editoriale.

6 L'ultima sezione, intitolata «Vies et pasque», si concentra sulle implicazioni della scelta autoriale di inscrivere la propria soggettività all'interno della narrazione, nonché, viceversa, su alcuni testi in cui lo scrittore appare soltanto come silhouette priva di spessore. Alain GOULET (Gide et ses alter ego expérimentaux, pp. 287-300) ripercorre l'evoluzione degli alter ego gidiani, immagini ipotetiche di sé attraverso le quali l'autore può vedersi agire a distanza e compiere una vera e propria catarsi; Paul DIRKX (Le corps autographe. Nouvelles hypothèses sur Le jardin des plantes de Claude Simon, pp. 301-312) si sofferma invece su Le jardin des plantes di Claude Simon per osservare come la presenza concreta del corpo-scrivente, il ricorso alla memoria e la creazione di una complessa rete di relazioni intertestuali lo rendano una archéo-logie réflexive capace di radicalizzare l'esplorazione della frontiera tra realtà e finzione. Il contributo di Robert DION e Frances FORTIER (Institutions en péril: de la satire dévastatrice à la disqualification parodique, pp. 313-328) esamina tre testi contemporanei nei quali la critica feroce ai meccanismi istituzionali di legittimazione letteraria si realizza anche attraverso la 
realizzazione di figure d'autori-fantoccio; al contrario, la sovra-esposizione di sé è programmaticamente dominante nella scrittura di Christine Angot, che Jacques DuBoIs (Devenir auteur: Angot actrice et témoin, pp. 329-338) legge attraverso la lente di un'arte contestuale volta a ristabilire il legame perduto tra produzione artistica e pubblico. Clotilde LANDAIS (La représentation littéraire de l'écrivain comme reflet de l'imposture de l'institution littéraire chez Stephen King, pp. 339-354) chiude la raccolta mostrando come la creazione di scrittori variamente impostori costituisca per Stephen King uno strumento per riposizionare la propria immagine, scardinando la divisione istituzionale tra letteratura "alta" e "sotto-letteratura" di genere.

7 L'interrogazione sulla referenzialità accomuna i diversi contributi ed è resa tanto più stimolante dalla scelta di considerare testi che mettono contemporaneamente in scena una molteplicità di attori del mondo letterario, introducendo uno spazio di ambiguità ancora da indagare. Il volume, che si annuncia come il prodotto di un chantier en pleine construction, non esita inoltre a suggerire molteplici aperture a nuovi sviluppi di ricerca. 